Rev Biomed 2006; 17:61-68.

\title{
Recomendaciones y fundamentos para la vacunación después de la esplenectomía por trauma en adultos. Una visión práctica.
}

\author{
Luis M. García-Núñez ${ }^{1}$, Ruy Cabello-Pasini²
}

${ }^{1}$ Cirujano General, International Visiting Scholar/Research - Fellow, Trauma Surgery and Surgical Critical Care, Department of Surgery - Division of Trauma, University Hospital, University of Medicine and Dentistry of New Jersey at Newark, Newark, New Jersey, US. ${ }^{2}$ Cirujano General y de Trauma, Médico Especialista Adjunto, Sala de Cirugía de Hombres, Hospital Central Militar, Secretaría de la Defensa Nacional, México, Distrito Federal, México.

\section{RESUMEN.}

Objetivo. Analizar las recomendaciones actuales de la practica de la vacunación en el paciente adulto esplenectomizado por trauma.

Fuentes y extracción de la información. Búsqueda bibliográfica y electrónica en la red de las recomendaciones actuales para la vacunación del paciente adulto esplenectomizado en trauma.

Resultados. Es bien conocido que el bazo juega un papel fundamental en la inmunidad contra las infecciones por gérmenes encapsulados. A pesar de que la practica actual apoya a las intervenciones de salvamento esplénico (incluyendo al manejo no operatorio), la esplenectomía esta indicada en ciertos casos. Estos pacientes son susceptibles a complicaciones sépticas por gérmenes encapsulados. Se han propuesto intervenciones profilácticas como la administración de antibióticos a largo plazo y la vacunación. Aunque el valor clínico de la vacunación es poco clara, ha mostrado su eficacia para incrementar los niveles de anticuerpos a títulos protectores.

Conclusiones. Para el paciente adulto esplenectomizado por trauma, las recomendaciones actuales de mayor nivel de evidencia medica sugieren la triple vacunación contra $S$. pneumoniae, $N$. meningitidis y Haemophilus influenzae tipo b al dia 14 postoperatorio, con revacunación a intervalos de entre 3 y 5 años (dependiendo del biológico específico). (Rev Biomed 2006; 17:61-68)

Palabras clave: esplenectomia, trauma, vacunación.

\section{SUMMARY.}

Recommendations and fundamentals for vaccination after splenectomy for trauma in adults. A practical viewpoint.

Objective. To analyze the current recommendations for the practice of vaccination in the traumasplenectomized adult patient.

Data-Source. Web-electronic and bibliographic

Solicitud de sobretiros: Mayor M.C. Luis M. Garcia-Núñez. Circuito Naranjeros No. 22, Colonia Club de Golf México, Tlalpan, Distrito Federal 14620, México. Teléfono 5573-0026, Fax 5573-0026.

Correo electrónico:Imgarcian@hotmail.com, luismanuelgarcianunez@yahoo.com

Recibido el 1/Septiembre/2005. Aceptado para publicación el 7/Noviembre/2005.

Este artículo está disponible en http://www.uady.mx/sitios/biomedic/revbiomed/pdf/rb061718.pdf 


\section{LM García-Núñez, R Cabello-Pasini.}

research of the current recommendations for vaccination in the trauma-splenectomized adult patient. Results. It is well known that the spleen plays a major role in immunity against infections due to capsulatedbacteria. Despite current practice supporting splenic salvage interventions (including non-operative management) splenectomy is indicated in some cases. These patients are susceptible to sustain septic complications secondary to capsulated-organisms. Prophylactic interventions such as long-term antibiotic administration and vaccination have been proposed. Although the clinical value of vaccination is unclear, it has shown its efficacy for increasing the levels of antibodies to protective titers.

Conclusions. For the trauma-splenectomized patient, current recommendations of the highest level of medical-evidence suggest triple-vaccination against $S$. pneumoniae, $N$. meningitidis and Haemophilus influenzae type b at $14^{\text {th }}$ postoperative day, and re-vaccination at intervals between 3-5 years (depending on the specific vaccine).

\section{(Rev Biomed 2006; 17:61-68)}

Key words: splenectomy, trauma, vaccination.

\section{INTRODUCCIÓN.}

Del 65 al 75\% de las lesiones esplénicas son secundarias a trauma contuso. Los mecanismos de lesión esplénica varían, siendo los accidentes automovilísticos aquellos que representan la mayor parte (1). Los mecanismos penetrantes origina alrededor de $20 \%$ de los casos de trauma esplénico; las heridas por proyectil de arma de fuego son la causa mas común en este subgrupo de lesiones $(2,3)$.

Las indicaciones de esplenectomía han cambiado dramáticamente con el tiempo. La intervenciones de salvamento esplénico se han incrementado importantemente en las últimas dos décadas, y éstas han evolucionado desde las maniobras quirúrgicas de conservación (uso de agentes hemostáticos, esplenorrafia, resección parcial), las cuales se han usado alternativamente a la esplenectomía, hasta el manejo no operatorio. El manejo no operatorio ha sido plenamente validado como conducta terapéutica bajo las condiciones que dictan los criterios propuestos por Wisner y Blaidsdell (4): 1) estabilidad hemodinámica, 2) hallazgos abdominales ausentes o minimos, 3) evidencia por laboratorio de pérdida hemática mínima, 4) mecanismo de trauma de baja energía, 5) determinación tomográfica de trauma esplénico aislado, 6) ausencia de lesiones intraabdominales asociadas, 7) ausencia de compromiso hiliar o disrupción masiva del bazo en la tomografía computada. Esta forma de manejo ha demostrado ser exitosa en $98 \%$ de los casos en adultos y en $83 \%$ en niños (1).

Sin embargo, la esplenectomía aún conserva su lugar; es de llamar la atención que Rose et al, en una estudio retrospectivo de la experiencia de 11 años en un Centro de Trauma Nivel I, señalan que el trauma es la indicación mas común para la extracción quirúrgica del bazo, y que $0.13 \%$ del total de pacientes que ingresan para cirugía por trauma, serán esplenectomizados (5).

El ímpetu en el salvamento esplénico esta basado en el reconocimiento que el bazo tiene una importante función en la respuesta del huésped a las infecciones, debido a su capacidad para destruir gérmenes encapsulados por polisacáridos (1). Esta respuesta involucra su remoción del torrente sanguíneo y la producción de anticuerpos específicos contra los antígenos polisacáridos. El bazo es el principal órgano involucrado en la fagocitosis de partículas con un bajo índice o ausencia de opsonización por C3b. Otra función importante del bazo es la producción de tuftsina, un tetrapéptido estimulador de la actividad fagocítica, generado por la ruptura enzimática de la cadena pesada de la molécula de IgG. Finalmente, el bazo provee de una reacción inmunológica primaria rápida por la producción de inmunoglobulinas después del contacto inicial con un antígeno, exponiendo a las células B de memoria productoras de anticuerpos a altas concentraciones de ese antígeno en el parénquima esplénico, ocasionando su eliminación (6,7). Se ha propuesto que la iniciación de una respuesta inmune primaria a los antigenos polisacáridos, incluyendo los neumocóccicos, está específicamente relacionada con

\section{Revista Biomédica}




\section{Vacunación y esplenectomia por trauma.}

el bazo, en particular con la zona marginal (6-8).

Después de la esplenectomía, hay un incremento en el riesgo del desarrollo de complicaciones sépticas, lo cual conlleva una alta mortalidad, principalmente en niños. Según Fielding (8), el riesgo de desarrollar una infección seria es de $5 \%$, y las infecciones fatales se presentan en $2 \%$ de los casos. Los organismos causales mas comunes de infecciones en el paciente esplenectomizado son $S$. pneumoniae, E. coli, $N$. meningitidis, $H$. influenzae, $P$. aeruginosa, Staphylococcus sp y otros estreptococos. Sin embargo, también se han reportado infecciones graves por Babesia sp, Capnocytophaga canimorsus (bacilo DF-2), Histoplasma sp y Plasmodium sp.

La manifestación séptica mas importante en el paciente esplenectomizado es el "síndrome de infección siderante post-esplenectomía" (OPSI por sus siglas en inglés). Este estado séptico se presenta en 0.5-2\% de los pacientes con extirpación quirúrgica del bazo y es causado generalmente por $S$. pneumoniae, seguido por $N$. meningitidis y $H$. influenzae. S. pneumoniae causa OPSI en el 50-90\% de los casos, y se han reportado varios serotipos (9-11). En 70\% de los casos, no hay evidencia de un foco claro de septicemia. Puede desarrollarse inmediatamente o tan tardíamente como a los 65 años post-esplenectomía. En el OPSI plenamente manifiesto, la mortalidad reportada es de $50-70 \%$ (9-12), a pesar de un tratamiento apropiado con antibióticos y apoyo en la Unidad de Cuidados Intensivos.

Por lo anteriormente expuesto, se han diseñado estrategias de prevención contra las complicaciones sépticas. Esta aceptado que la esplenectomía es una indicación para la vacunación contra gérmenes encapsulados. Aunque algunos estudios han sugerido que los pacientes asplénicos tienen una respuesta subnormal a las vacunas, existe algún grado de respuesta en prácticamente la totalidad de los mismos (13). Desde este punto de vista, la vacuna mejor estudiada ha sido la neumocóccica. Otras vacunas, como la fabricada para inmunizar contra infecciones secundarias a Haemophilus y Meningococcus han sido evaluadas con menos detalle, pero se sabe que son inmunogénicas. La administración simultánea de la triple vacunación ha demostrado su seguridad, efectividad e inmunogenicidad en adultos por otro lado sanos.

Desafortunadamente, el OPSI también ha sido reportado en pacientes vacunados. Debido a eso, en adición a la vacunación, varios autores han recomendado la profilaxis antibiótica indefinida con penicilínicos (amoxicilina $250 \mathrm{mg} /$ día) o eritromicina. Chattopadway (13) recomienda un esquema de 10 años, mientras que Waghorn (14) señala que estos deben ser administrados durante los primeros dos años post-esplenectomía. Machesky y Cushing (15), en una revisión de la literatura, señalan que la mayoría de los autores recomienda profilaxis hasta los 18 años en niños, y por lo menos durante 5 años en los adultos.

\section{VACUNACIÓN DEL PACIENTE ASPLÉNICO.}

Como se mencionó con anterioridad, los pacientes esplenectomizados deben vacunarse para disminuir la probabilidad de complicaciones sépticas (13-15). La cobertura mínima que debe contemplarse en el esquema de inmunización es contra Streptococcus pneumoniae, Haemophilus influenzae tipo b y Neisseria meningitidis (16).

\section{Características de las vacunas disponibles.}

Vacuna neumocóccica polivalente (Pneumovax 23 ${ }^{\circledR}$, Merck \& Co; Inc. Whitehouse Station, NJ, USA): La vacuna antineumocóccica es una vacuna polivalente que contiene 25 microgramos de polisacárido capsular purificado de cada uno de los 23 serotipos capsulares de neumococos mas prevalentes en los Estados Unidos, incluyendo los seis serotipos que mas frecuentemente causan infecciones invasivas fármaco-resistentes, y los cuales se presentan en $85-90 \%$ de los casos de infección postesplenectomía. Los anticuerpos generados por la vacuna declinan a los 5 años post-inmunización, y la revacunación se recomienda a los individuos con asplenia post-quirúrgica, ya que los anticuerpos decaen aun con mayor rapidez (16). Hosea et al (17), por su parte, demostraron que la respuesta a la vacuna es sub-óptima en términos de titulación absoluta de los anticuerpos generados, incremento relativo y tasa de incremento de los mismos. La respuesta a los 


\section{LM García-Núñez, R Cabello-Pasini.}

anticuerpos parece ser aún mas precaria en los pacientes con malignidad hematológica asociada $o$ bajo quimioterapia (8). La vacuna se administra en una sola dosis de $0.5 \mathrm{ml}$, subcutánea; los niveles protectores de anticuerpos se obtienen alrededor de la tercera semana post-vacunación. La revacunación después de 5 años se encuentra justificada (16).

Se presenta en viales de producto biológico en forma líquida $(16,18)$, para administrar 5 o 10 dosis sin necesidad de reconstitución.

Entre los efectos adversos de la vacuna (18) figuran reacciones locales como adormecimiento, eritema, inflamación local e induración indolora; la celulitis es rara pero se ha reportado como un fenómeno pasajero alrededor del 2/o día postvacunación. Las reacciones sistémicas consisten en fiebre, malestar general, astenia, náusea, vómito, linfadenitis, trombocitopenia, anemia hemolítica, anafilaxia, enfermedad del suero, edema angioneurótico, mioartralgias, cefalea, parestesias, radiculoneuropatía, síndrome de Guillain-Barré, exantema y urticaria.

Según las últimas recomendaciones farmacológicas, la única contraindicación para la aplicación de la vacuna es la hipersensibilidad conocida a los componentes de la fórmula (18). Debe aplicarse con precaución a pacientes con función cardíaca o pulmonar seriamente comprometida. No existen estudios acerca de la plena seguridad de la vacuna neumocóccica en el embarazo; por tanto, solo debe administrarse a pacientes embarazadas si está claramente indicado. Tampoco se conoce la seguridad del uso de la vacuna durante la lactancia. En esta circunstancia, debe administrarse con suma precaución.

La respuesta inmunológica en niños menores de dos años es impredecible (16-18), y debido a esto, a pesar de que no está contraindicada, no se recomienda en este grupo etario. Para este grupo de pacientes, el Southern Health Board del Reino Unido (19) ha propuesto el uso de un conjugado heptavalente que esta aprobado para su uso en niños menores de 2 anos de edad con factores de riesgo. Este biológico confiere inmunidad contra $70 \%$ de los organismos que causan complicaciones sépticas invasivas en los pacientes esplenectomizados.

Como se mencionó previamente, hay reportes de OPSI a pesar de la vacunación, y no está claro si los pacientes que sucumbieron a esta entidad tuvieron una respuesta adecuada a la vacunación. Golemathis et al (20) señala que la vacunación asociada a la administración de penicilinicos es una estrategia confiable para prevenir la OPSI.

Vacuna meningocóccica (Menomune - A/C/ Y/W-135 ${ }^{\circledR}$, Aventis Pasteur Inc. Swiftwater PA, USA): Esta vacuna consiste en un extracto purificado liofilizado del polisacárido capsular externo de Neisseria meningitidis, la cual es efectiva contra los serogrupos A, C, Y y W-135 (16). No hay vacuna efectiva contra el serogrupo B (21). La respuesta serológica a la vacuna es adecuada en $90 \%$ de los individuos sanos a los cuales se aplica. Se recomienda una dosis única de $0.5 \mathrm{ml}$ por vía subcutánea. $\mathrm{Su}$ presentación consiste en viales de diluyente de 0.78 $\mathrm{ml}$ (sin preservativo), $1 \mathrm{ml}$ (sin preservativo) o $6 \mathrm{ml}$ (con preservativo) para reconstituir el liofilizado y administrarlo con jeringa estéril como está recomendado (22).

Entre los pacientes con esplenectomía por trauma, la proporción de seroconversión no difiere de los controles, pero no hay un pico de producción de IgM. Sin embargo, la respuesta se traduce claramente en un beneficio clínico. Los niveles protectores de anticuerpos se obtienen de 7 a 10 días después de la vacunación. La revacunación se recomienda en los pacientes esplenectomizados, por considerarse un grupo de alto riesgo (21).

Las reacciones adversas mas comunes consisten en dolor y enrojecimiento en el sitio de la inyección por uno o dos días. Se han reportado también fiebre, malestar general, cefalea, reacciones de hipersensibilidad y nefropatía por IgA (21).

La vacuna se encuentra contraindicada durante el curso de una enfermedad aguda y en pacientes con hipersensibilidad conocida al timerosal o a cualquier otro componente de la vacuna. No debe administrase en conjunto con la inmunización contra Pertussis debido a la combinación de la carga de endotoxina.

\section{Revista Biomédica}


Vacunación y esplenectomia por trauma.

No existe contraindicación alguna para su aplicación a menores de dos años, pero tampoco existen recomendaciones claras para su administración; por tanto, no se recomienda para este grupo erario $(21,22)$.

Vacuna anti-Haemophilus influenzae $B$ (Hib)(Haemophilus Conjugate Vaccine [Diphteria CRM ${ }_{197}$ Protein Conjugate] HibTITER, Wyeth Pharmaceuticals Inc. Philadelphia PA, USA): Esta vacuna consiste en un conjugado de oligosacáridos del antígeno capsular de $H$. influenzae tipo b (Haemophilus b) y la proteína diftérica $\mathrm{CRM}_{197}$ $\left(\mathrm{CRM}_{197}\right)$, para promover su inmunogenicidad (23). Noventa a noventa y nueve por ciento de los individuos sanos responden con niveles protectores de anticuerpos después de 3 dosis. Hay datos que sugieren que los individuos asplénicos pueden responder a la vacunación y desarrollar niveles adecuados de anticuerpos aunque una vez mas, la eficacia protectora de la vacuna no se ha confirmado clínicamente.

El curso primario de inmunización consiste en 3 dosis de $0.5 \mathrm{ml}$ por vía intramuscular. La vacuna se presenta en forma de viales de $0.5 \mathrm{ml}$ de biológico líquido, sin necesidad de reconstitución $(16,23)$.

Entre los efectos adversos reportados se encuentran fiebre, eritema local, inflamación en el sitio de vacunación, irritabilidad, letargia, somnolencia, malestar general y llanto inconsolable, pérdida del apetito, diarrea, exantema, urticaria, decoloración de la piel, anafilaxia, edema angioneurótico, convulsiones, eritema multiforme, edema facial, síndrome de GuillainBarré, cefalea y síndrome de hiporrespuesta hipotónica episódica. En pacientes en los cuales la inmunización se ha aplicado en conjunto con las vacunas DTP, DtaP, hepatitis B, IPV, OPV, neumocóccica septavalente, MMR y/o meningocóccica del grupo C (23), se ha reportado apnea temporal.

La vacuna se encuentra contraindicada en pacientes con hipersensibilidad conocida a cualquier componente de la fórmula, incluyendo el toxoide diftérico. Debe emplearse con precaución en pacientes con eventos previos de anafilaxia tras inmunizaciones, en pacientes con alteraciones adquiridas en la respuesta inmune (terapia inmunosupresora, irradiación, corticosteroides, antimetabolitos, agentes alquilantes y citotóxicos)(8), defectos genéticos, infección por el virus de la inmunodeficiencia humana, enfermedad febril aguda de cualquier índole y pacientes anticoagulados (11). No ha sido evaluada para determinar sus efectos sobre la carcinogénesis, mutagénesis o alteraciones en la fertilidad. No está recomendada para usarse rutinariamente en la población adulta, en pacientes embarazadas o en menores de 6 semanas de edad (23).

\section{Aplicación de la vacunación.}

Con base en lo previamente expuesto, el Departamento de Educación Quirúrgica del Centro Médico Regional de Orlando ha desarrollado las siguientes recomendaciones prácticas para la vacunación del paciente esplenectomizado por trauma. Dichas recomendaciones se encuentran fundamentadas bajo los principios de los lineamientos de "Medicina Basada en la Evidencia" (16) (ver cuadro 1):

A. Los pacientes esplenectomizados de manera no electiva deben vacunarse en el día 14 postoperatorio (Recomendación nivel 1).

B. Los pacientes asplénicos deben ser revacunados a intervalos apropiados de tiempo (ver recomendaciones próximas en el texto)(Recomendación nivel 2).

C. Los pacientes inmunocomprometidos deben ser vacunados al momento en el que el diagnóstico de inmunocompromiso se define (Recomendación nivel $3)$.

D. Cuando la vacunación en adultos está indicada, deben administrarse los siguientes biológicos (Recomendación nivel 3):

- Vacuna neumocóccica polivalente (Pneumovax $23^{\circledR}$, Merck \& Co; Inc. Whitehouse Station, NJ, USA): una dosis de $0.5 \mathrm{~mL}$ subcutánea en la región deltoidea o lateral del muslo al día 14 postoperatorio, y revacunar cada 5-6 años.

- Vacuna de polisacárido meningocóccico cuadrivalente (Menomune - A/C/Y/W-135 ${ }^{\circledR}$, Aventis Pasteur Inc. Swiftwater PA, USA): una dosis de $0.5 \mathrm{~mL}$ subcutánea en la región deltoidea o lateral del muslo al día 14 postoperatorio, y revacunar cada 


\section{LM García-Núñez, R Cabello-Pasini.}

\section{Cuadro 1}

Clases de estudios y nivel de recomendaciones que fundamentan la evidencia en Medicina (16).

Estudio de clase I: Estudios controlados, prospectivos y randomizados, son el estándar de oro de los estudios clínicos.

Estudios de clase II: Estudios en los cuales los datos se colectan prospectivamente, o aquellos en los cuales se efectuaron estudios retrospectivos basados en datos claramente confiables (estudios observacionales, estudios de cohorte, estudios de prevalencia, o estudios de casos control.

Estudios de clase III: Estudios basados en datos colectados retrospectivamente (series clínicas, revisión de registros o bases de datos, revisión de grandes series de casos y opiniones de expertos).

La evaluación de los avances de la tecnología no se encuentra contemplada en el formato de la clasificación previamente mencionada.

Recomendación de nivel 1. Este tipo de recomendaciones es justificable basada solamente en la información científica disponible. El fundamento de las recomendaciones de nivel 1 son estudios de clase I, aunque ocasionalmente algunos estudios de clase II pueden formar parte de ellas.

Recomendación de nivel 2. Este tipo de recomendación es razonablemente justificable por la evidencia científica disponible y fuertemente apoyada por las opiniones de expertos. Usualmente esta fundamentada en estudios de clase II o una preponderancia de evidencia de clase III.

Recomendación de nivel 3. Esta recomendación esa fundamentada en datos disponibles en la literatura, pero no representan una evidencia científica validada. Generalmente se encuentra sustentada en datos de clase III. Es útil con propósitos educacionales y como guía para futuras investigaciones.

3 (10) a 5 años (16).

- Vacuna conjugada de Haemophilus b (Haemophilus Conjugate Vaccine [Diphteria CRM $_{197}$ Protein Conjugate] HibTITER, Wyeth Pharmaceuticals Inc. Philadelphia PA, USA): una dosis de $0.5 \mathrm{~mL}$ intramuscular en la región deltoidea o lateral del muslo al día 14 postoperatorio, no hay necesidad de revacunar.

E. La vacunación pediátrica debe efectuarse de acuerdo a las dosis y esquemas pediátricos recomendados, con especial consideración a los niños menores de 2 años de edad (Recomendación nivel 3).

\section{DISCUSIÓN.}

Como se ha notado, los fundamentos de las recomendaciones actuales para la vacunación en el paciente adulto esplenectomizado debido a una lesión traumática distan mucho de ser sólidas. Se calcula que solo $37.5 \%$ de los pacientes en los cuales el bazo se extirpo quirúrgicamente por una lesión traumática, son vacunados bajo lineamientos basados en la evidencia medica (14). Según lo establecido por el Departamento de Educación Quirúrgica del Centro Medico Regional de Orlando (16), solo dos estudios Revista Biomédica recientes de clase I han demostrado puntos validados. Uno de ellos es que los títulos de anticuerpos que obtienen tras la vacunación polivalente neumocóccica alcanzan una cifra optima cuando este biológico se aplica en el dia 14 postoperatorio, ya que determinaciones seriadas de anticuerpos en pacientes esplenectomizados que recibieron la vacunación neumocóccica en los días 1,7 y 14 postoperatorios, han demostrado que los títulos obtenidos con la administración del agente a este tiempo genera los niveles de anticuerpos mas altos. El otro nos evidencia que desafortunadamente, la inmunidad conferida por la vacunación no previene completamente la aparición de OPSI.

Contamos, sin embargo, con un cúmulo mayor de datos de clase II o III (16). Dentro de estas evidencias, encontramos que esta demostrado el bazo juega un papel fundamental en la inmunidad contra infecciones por gérmenes encapsulados. En este mismo estrato de evidencia, también hemos observado que las recomendaciones actuales de los Centros de Control de Enfermedades de los Estados Unidos de América (Center of Disease Control - CDC por sus siglas en ingles) señalan que en los pacientes esplenectomizados es requerida la triple vacunación 


\section{Vacunación y esplenectomia por trauma.}

(neumocóccica polivalente, meningocóccica cuadrivalente y contra Haemophilus influenzae tipo b) (16). Las recomendaciones relativas a la revacunación aún se encuentra clasificadas dentro del nivel 2 y deberan ser validadas con el tiempo.

Los lineamientos de la práctica de la administración de la triple vacunación en el paciente adulto esplenectomizado se encuentran fundamentados en la farmacopea actual, y derivan mayormente de la experiencia en su aplicación a la población general $(16,18,21,23)$.

No existen estudios de clase I que demuestren el verdadero impacto clinico de la vacunación en el paciente esplenectomizado por trauma $(3,7)$. Los estudios serologicos de inmunidad efectuados en los pacientes asplénicos vacunados evidencian que las determinaciones de anticuerpos contra los gérmenes encapsulados muestran títulos protectores a las 2-3 semanas post-intervencion, y que los títulos protectores se sostendrán con la revacunación a intervalos regulares. Debido a eso, se considera que el impacto epidemiológico es contundente.

\section{CONCLUSIONES.}

Los lectores de esta revisión deben estar de que la vacunación de los pacientes esplenectomizados por trauma es un área en la cual se están tratando de establecer concensos. Como se mencionó anteriormente, la evidencia de que el paciente esplenectomizado es mas susceptible que la población general a complicaciones sépticas devastadoras por gérmenes encapsulados es incuestionable. Los cirujanos de trauma que practican esta intervención quirúrgica no pierden de vista que la vacunación siguiente al procedimiento operatorio es obligada (24). El problema actual es que los lineamientos carecen de soporte por recomendaciones de nivel 1 , siendo la mayoría de estas provenientes de recomendaciones de nivel 2 o 3.

El impacto clinico de la vacunación es desconocido $(3,7)$, sin embargo, los estudios serológicos de inmunidad en grandes series de pacientes esplenectomizados por trauma, demuestran un contundente incremento de los niveles de anticuerpos, los cuales observan títulos protectores, siendo capaces de mantenerse en este rango por vía de la revacunación. Esto, desafortunadamente, no previene de manera absoluta la posibilidad de presentar OPSI.

Solo los hechos y la práctica rutinaria adicional de la vacunación en el paciente esplenectomizado proveerán de datos sostengan una mayor cantidad de recomendaciones de nivel 1 y que hagan de esta una intervención consistente y racional. Saffle (24) recomienda que la comunidad de cirujanos de trauma debe empezar desde luego a recolectar los datos multicéntricos requeridos para elevar su nivel cultural y lidiar con los prejuicios que previenen la existencia de lineamientos correctos.

\section{REFERENCIAS.}

1.- Gangemi JJ, Binns OA, Young JS. Splenic injury after athtetic trauma: a case report of splenic rupture induced by Lacrosse Ball. J Trauma 1999; 46:736-7.

2.- Garcia F, Arango LA, Angel A. Trauma esplénico. Estudio retrospectivo en 9 años (1982-1990). Rev Col Cirugía 1994; 9:33-6.

3.- Sixto DA, Sixto IB. La esplenectomía segmentaria sobrepasa los pronósticos. Rev Cub Cir 2003; 42:45-53.

4.- Wisner DH, Blaidsdell FW. When to save the ruptured spleen. Surgery 1992;111:121-2.

5.- Rose AT, Newman MI, Debelak J, Pinson CW, Morris JA $\mathrm{Jr}$, Harley DD, et al. The incidence of splenectomy is decreasing: lessons learned from trauma experience. Am Surg 2000; 66:481-6.

6.- Zoli G, Corazza GR, Wood S, Bartoli R, Gasbarrini G, Farthing MJG. Impaired splenic function and tuftsin deficiency in patients with intestinal failure on long term intravenous nutrition. Gut 1998; 43:759-62.

7.- Leemans R, Manson W, Snijder JAM, Smit JW, Klasen $\mathrm{HJ}$, The T, et al. Immune response capacity after human splenic autotransplantation. Ann Surg 1999; 229(2):279-85. 8.- Fielding AK. Prophylaxis against late infection following splenectomy and bone marrow transplant. Blood Reviews 1994; 8:179-91.

9.- Remouñan BC, Sanchez GC, Vázquez VL, Davas SR, Reyes PM, Valdés GMC. Fallo multiorgánico secundario a sepsis

Vol. 17/No. 1/Enero-Marzo, 2006 


\section{LM García-Núñez, R Cabello-Pasini.}

post-esplenectomía. Presentación de un caso. Rev Cubana Med 1999; 38:67-70.

10.- Shatz DV. Vaccination practices among North American trauma surgeons in splenectomy for trauma. J Trauma 2002; 53:950-6.

11.- Waghorn DJ. Overwhelming infection in asplenic patients: current best practice preventive measures are not being followed. J Clin Pathol 2001; 54:214-8.

12.- Locker GL, Wagner A, Peter A, Staudinger T, Marosi C, Rintelen C, et al. Lethal Waterhouse-Friderichsen syndrome in posttraumatic asplenia. J Trauma 1995; 39:784-6.

13.- Chattopadway B. Splenectomy, pneumococcal vaccination and antibiotic prophylaxis. Br J Hosp Med 1989; 41:172-4.

14.- Waghorn DJ. Prevention of post-splenectomy sepsis. Lancet 1993; 341:248-9.

15.- Machesky KK, Cushing RD. Overwhelming postsplenectomy infection in a patient with penicillin-resistant Streptococcus pneumoniae. Arch Fam Med 1998; 7:178-80.

16.- Department of Surgical Education - Orlando Regional Medical Center. Splenic vaccine prophylaxis. Practical Guidelines [serial on line] 2003 January [cited 2003 April 24]; 1 (1): [4 screens] Available from URL: http:// www.surgicalcriticalcare.net/Guidelines/PDF/ splenicvaccineprophylaxis.pdf.

17.- Hosea SW, Burch CG, Brown EJ, Berg RA, Frank MM. Impaired response of splenectomized patients to polyvalent pneumococcal vaccine. Lancet 1981; 11:804-7.

18.- Pharmacologic description - Pneumovax 23 (Pneumococcal vaccine polyvalent). [serial on line] 1986 January-March [cited 2005 August 4];1(1):[8 screens] Available from:URL: www.merck.com/product/usa/ pi circulars/pneumovax 23/pneumovax pi pdf-.

19.- Southern Health Board. Guidelines for the prevention and treatment of infection in patients with absent or dysfunctional spleen. Department of Public Health and Infectious Diseases - Southern Health Board J 2002; 1:1-8.

20.- Golemathis B, Tzardis P, Legakis N, Persidou-Golemati P. Report about the efficacy of anti-pneumococcal vaccination and antibiotic prophylaxis in asplenic patients. Mt Sinai J Med 1989; 56:97-8.
21.- AHFS. Meningococcal Polysaccharide Vaccine Groups A,C, Y and W-135 Combined - Menomune - A/C/Y/W-135. [serial on line] 2003 January [cited 2005 June 16];1(1):[5 screens]. Available from: URL: www.vaccinesshope.com/ us pdf/menomune 4813.487512 .03 pdf-.

22.- Melton KA. Pneumococcal vaccine. Prim Care Update Ob/Gyns 2001; 8:44-7.

23.- Recommendation W10461C004 - Wyeth Pharmaceuticals. Haemophilus $b$ conjugate vaccine (Diphteria $\mathrm{CRM}_{197}$ Protein Conjugate) HibTITER®. [serial on line] 2002 March-April [cited 2005 May 12];1(1):[31 screens]. Available from: www.vaccinesshope.com/ us pdf/menomune $4813.4875 \quad 12.03$ pdf-.

24.- Saffle JR. A comprehensive practice guideline is needed to make vaccination practices more consistent. J Trauma 2002; 53:1026-7.

\section{Revista Biomédica}

